\title{
Molecular classification of spontaneous endometrial adenocarcinomas in BDII rats
}

\author{
Emma Samuelson $^{1,2}$, Carola Hedberg ${ }^{1,2}$, Staffan Nilsson $^{3}$ and Afrouz Behboudi ${ }^{1}$
}

${ }^{1}$ Department of Medical and Clinical Genetics, Institute of Biomedicine, University of Gothenburg, Sahlgrenska Academy, SE 40530 Gothenburg, Sweden

${ }^{2}$ Department of Cell and Molecular Biology, Genetics, Lundberg Laboratory, University of Gothenburg, SE 40530 Gothenburg, Sweden

${ }^{3}$ Department of Mathematical Statistics, Chalmers University of Technology, Gothenburg, Sweden

(Correspondence should be addressed to A Behboudi; Email: afrouz.behboudi@gu.se)

\begin{abstract}
Female rats of the BDII/Han inbred strain are prone to spontaneously develop endometrial carcinomas (EC) that in cell biology and pathogenesis are very similar to those of human. Human EC are classified into two major groups: Type I displays endometroid histology, is hormone-dependent, and characterized by frequent microsatellite instability and PTEN, K-RAS, and CTNNB1 ( $\beta$-Catenin) mutations; Type II shows non-endometrioid histology, is hormone-unrelated, displays recurrent TP53 mutation, CDKN2A (P16) inactivation, over-expression of ERBB2 (Her2/neu), and reduced $\mathrm{CDH} 1$ (Cadherin 1 or E-Cadherin) expression. However, many human EC have overlapping clinical, morphologic, immunohistochemical, and molecular features of types I and II. The EC developed in BDII rats can be related to type I tumors, since they are hormone-related and histologically from endometrioid type. Here, we combined gene sequencing (Pten, Ifr1, and Ctnnb1) and real-time gene expression analysis (Pten, Cdh1, P16, Erbb2, Ctnnb1, Tp53, and Irf1) to further characterize molecular alterations in this tumor model with respect to different subtypes of EC in humans. No mutation in Pten and Ctnnb1 was detected, whereas three tumors displayed sequence aberrations of the Irf1 gene. Significant down regulation of Pten, Cdh1, p16, Erbb2, and Ctnnb1 gene products was found in the tumors. In conclusion, our data suggest that molecular features of spontaneous EC in BDII rats can be related to higher-grade human type I tumors and thus, this model represents an excellent experimental tool for research on this malignancy in human.
\end{abstract}

Endocrine-Related Cancer (2009) 16 99-111

\section{Introduction}

Endometrial cancer is the most frequently diagnosed gynecological malignancy in the western world with an estimated incidence of 15-20 cases per 100000 women per year (Esteller et al. 1999, Ryan et al. 2005). Endometrial carcinoma (EC) is the prevalent subtype, accounting for $\sim 75 \%$ of the reported cases (Cavanagh et al. 1999). Most of the ECs are sporadic, but an inherited genetic predisposition has been demonstrated in about 5\% of cases, as the risk for a woman to develop EC is tripled when there is an affected first-degree relative (Sandles et al. 1992, Gruber \& Thompson 1996, Sandles 1998). Based on the biology and clinical features, two different pathways are distinguished for tumorigenesis of sporadic EC, $\sim 80 \%$ are designated as type I, including those with endometrioid histology, mostly of low grade, occur between the ages of 20-54 years old, follow the estrogen-related pathway, and have a favorable outcome. Type II tumors comprise about $10-20 \%$ of sporadic cases, show non-endometrioid histology (usually papillary serous or clear cell, arising occasionally in endometrial polyps or from precancerous lesions that develop in atrophic endometrial), usually occur at latter age (5-10 years later than type I tumors). This type follows the estrogen-unrelated pathway and is characterized by an aggressive clinical course and poor prognosis (Lax 2004, 2007, Ryan et al. 2005, Hecht \& Mutter 2006, Liu 2007). Using molecular analysis, these two pathways are further characterized by distinctive types of genetic instability and molecular alterations, including frequent microsatellite instability accompanied by PTEN, K-RAS, and CTNNB1 ( $\beta$-Catenin) mutations in type I, and TP53 
mutation, $C D K N 2 a(P 16)$ inactivation, overexpression of ERBB2 (Her2/neu), and reduced $C D H 1$ (Cadherin 1 or E-Cadherin) expression in type II tumors (reviewed in (Lax 2007, Liu 2007) and summarized in Table 4). However, the boundary between the two types is blurry. In fact, there is evidence for a third and larger group of EC with overlapping clinical and molecular features of both types I and II endometrial cancers. For instance, many of low-grade type I endometrioid carcinoma occur unrelated to estrogen and are found in the background of atrophic endometrium. On the other hand, it has been clearly shown that papillary serous carcinomas may occasionally develop from a pre-existing endometrioid carcinoma as a result of tumor progression, giving rise to mixed endometrioid-non-endometrioid carcinomas (Carcangiu \& Chambers 1992, Catasus et al. 1998). Accordingly, these tumors may share the pathologic and molecular features of types I and II endometrial cancers.

The genetic events underlying susceptibility to hereditary endometrial cancer are still poorly understood. Familial clustering of EC is reported to be the most common extra-colonic malignancy in hereditary non-polyposis colorectal cancer (HNPCC), a multi organ cancer syndrome with mismatch repair (MMR) deficiency (Miyaki et al. 1997, Peltomaki \& Vasen 1997, Lynch \& de la Chapelle 1999, Ollikainen et al. 2005). This group of EC are related to type I tumors, since they occur at young age and are histologically of mucinous or endometrioid type (Parc et al. 2000), but their pathway is driven by germ line mutations and is, thus, distinctive (Liu 2007). In addition to endometrial cancer arising from HNPCC, occasional families show clustering of endometrial cancer alone, without colon or other cancers. This group was termed as familial sitespecific endometrial cancer (Sandles 1998). Inactivation of MMR seems not to be of central role in tumorigenesis of these tumors (Table 4), suggesting another as yet unknown etiology in the most familial site-specific endometrial cancer (Ollikainen et al. 2005).

It appears that much work still remains to be done to clearly understand the biologic processes behind the development of different groups of EC. For this purpose, an animal model would be convenient to use. Females of the inbred BDII/Han (here after BDII) rat strain are genetically prone to spontaneously develop EC (Deerberg \& Kaspareit 1987, KaspareitRittinghausen et al. 1987). These tumors display large similarities in cell biology and pathogenesis to those of human (Deerberg \& Kaspareit 1987). Endometrial carcinogenesis in female BDII rats is hormone dependent (Deerberg \& Kaspareit 1987, Deerberg et al. 1995) and are the only early-onset tumors in the animals (Kaspareit-Rittinghausen et al. 1987). Thus, in terms of an endometrial cancer model, the female rats of this strain are unique (Vollmer 2003). Over the past decade, we have subjected this model to genetic characterizations and in the present study, we combined gene expression, allelic imbalance (AI), and mutation studies to investigate the extent of similarity of tumors developed in the BDII model to those of human EC. We investigated the expression levels of Pten, Tp53, Irf1, Ctnnb1 ( $\beta$-catenin), Cdkn2a (P16), Cdh1 (Cadherin 1, E-cadherin), and Erbb2 (Her2/neu) in rat endometrial adenocarcinoma compared with nonmalignant endometrium (NME) in quantitative realtime PCR. A selected number of tumors were screened for mutations in the Pten, Irfl, and Ctnnb1 genes using direct sequencing as well as for (AI, sometimes referred to as LOH analysis) at the Pten locus.

\section{Materials and methods}

\section{Animal crosses and tumor material}

Animals of the inbred BDII rat strain are genetically predisposed to spontaneous endometrial adenocarcinoma (EAC), with an incidence of more than $90 \%$ in virgin females as early as 13 months of age (Deerberg \& Kaspareit 1987, Kaspareit-Rittinghausen et al. 1987). BDII females were crossed to males from two non-susceptible strains, BN/Han, and SPRD-Cu3/Han. The F1-animals were crossed to each other or crossed back to BDII females to generate F2 and backcross (N1) populations. Spontaneously arising tumors developed in a proportion of F1, F2, and N1 animals. Tumors in the progeny were pathologically classified mainly as EAC or other uterine tumors (Roshani et al. 2001, 2005). In some cases, no malignant cells were detected in the removed cell mass when pathologically characterized. However, numerical chromosomal changes were detected in these samples (unpublished data). We consider these tissues represent normal or pre-malignant endometrium and are therefore of great importance in the present study. Herein, these cell lines are referred to as NME samples. At necropsy, tumor specimens and matched liver and/or spleen tissue samples were collected from animals for DNA extraction and cell culture establishment (Helou et al. 2001). Small pieces of fresh tumor tissues were used to set up primary cell cultures and a total of 40 cultures were established, representing 33 EAC tumors and seven NME samples (Table 1). A panel of 29 EAC and seven NME samples were selected for quantitative Real-time PCR analysis. Based on the results, we 
Table 1 Panel of cell cultures for the present study

\begin{tabular}{|c|c|c|c|c|}
\hline Cross & Generation $^{a}$ & Tumor designation & Tumor type $^{b}$ & Character number ${ }^{\mathrm{C}}$ \\
\hline \multirow[t]{24}{*}{ BDIIxBN } & $\mathrm{F} 1$ & RUT7 & EAC & 43 \\
\hline & $\mathrm{F} 1$ & RUT12 & $\mathrm{EAC}$ & 51 \\
\hline & $\mathrm{F} 2$ & RUT25 & EAC & 48 \\
\hline & $\mathrm{F} 2$ & RUT30 & EAC & 61 \\
\hline & N1 & NUT6 & EAC & 60 \\
\hline & N1 & NUT16 & EAC & 42 \\
\hline & $\mathrm{N} 1$ & NUT51 & EAC & 71 \\
\hline & N1 & NUT52 & EAC & 42 \\
\hline & N1 & NUT81 & EAC & 42 \\
\hline & $\mathrm{N} 1$ & NUT97 & EAC & 52 \\
\hline & N1 & NUT99 & EAC & 67 \\
\hline & N1 & NUT100 & EAC & 67 \\
\hline & $\mathrm{N} 1$ & NUT127 & EAC & 70 \\
\hline & N1 & NUT128 & EAC & 64 \\
\hline & $\mathrm{N} 1$ & NUT31 & EAC & 67 \\
\hline & $\mathrm{N} 1$ & NUT46 & EAC & 56 \\
\hline & N1 & NUT50 & EAC & 64 \\
\hline & $\mathrm{N} 1$ & NUT76 & EAC & 43 \\
\hline & $\mathrm{N} 1$ & NUT82 & EAC & 78 \\
\hline & N1 & NUT98 & EAC & 52 \\
\hline & N1 & NUT118 & NME & nd \\
\hline & N1 & NUT122 & NME & nd \\
\hline & N1 & NUT123 & NME & nd \\
\hline & $\mathrm{N} 1$ & NUT129 & NME & nd \\
\hline \multirow[t]{16}{*}{ BDIIxSPRD } & $\mathrm{F} 1$ & RUT2 & EAC & 38 \\
\hline & $\mathrm{F} 2$ & RUT3 & EAC & 59 \\
\hline & $\mathrm{F} 2$ & RUT6 & EAC & 58 \\
\hline & $\mathrm{F} 2$ & RUT13 & EAC & 62 \\
\hline & N1 & NUT4 & EAC & 62 \\
\hline & N1 & NUT7 & EAC & $46^{*}$ \\
\hline & N1 & NUT12 & EAC & 59 \\
\hline & N1 & NUT47 & EAC & 48 \\
\hline & N1 & NUT55 & EAC & 44 \\
\hline & N1 & NUT84 & EAC & $41^{*}$ \\
\hline & N1 & NUT39 & EAC & 39 \\
\hline & $\mathrm{N} 1$ & NUT42 & EAC & 44 \\
\hline & N1 & NUT14 & EAC & 44 \\
\hline & N1 & NUT89 & NME & nd \\
\hline & N1 & NUT58 & NME & nd \\
\hline & N1 & NUT18 & NME & nd \\
\hline
\end{tabular}

Tumors selected for gene mutation sequencing are shaded grey. *Additional tetraploid subpopulation found in the tumors.

${ }^{\mathrm{a}} \mathrm{F} 1$, strain intercross first generation animals; F2, intercross second generation animal; N1 = F1 animal back-crossed to BDII female.

${ }^{b} \mathrm{EAC}$, endometrial adenocarcinoma; NME, non-malignant endometrium.

${ }^{\mathrm{c}}$ Average of chromosome number in tumor.

selected a second panel of 14 EAC tumors for mutation sequencing (Ctnnbl, Ifrl, and Pten) as well as AI analysis of Pten (Table 1).

\section{Real-time quantitative PCR}

Expression of seven genes (Pten, Ctnnb1, P16, Tp53, Erbb2, Irfl, and $C d h 1$ ) was analyzed in 36 samples using quantitative Real-time PCR.

\section{cDNA preparation}

Two microgram total RNA of each sample was treated with DNaseI (Life Technologies) for $15 \mathrm{~min}$ at room temperature to remove any trace of genomic DNA; DNaseI was inactivated by adding Stop buffer and incubated at $70{ }^{\circ} \mathrm{C}$ for $10 \mathrm{~min}$. The treated RNA was then reverse-transcribed in reactions containing, Buffer RT, Oligo-dT primer, dNTP-mix, RNase inhibitor, and Ominiscript Reverse Transcriptase at $37^{\circ} \mathrm{C}$ for $1 \mathrm{~h}$ 
according to the protocol provided by manufacturer (Qiagen).

\section{Endogenous control}

We used data from an earlier cDNA oligo array analysis (Karlsson et al. 2007) on this material to select the most appropriate endogenous controls, (i.e. Gapdh (glyceraldehyde-3-phosphate dehydrogenase), $\beta$-actin, and Rps9 (ribosomal protein S9)), for the Real-time PCR quantification analysis.

\section{Real-time quantitative PCR with TaqMan}

Real-time PCR was performed in 384-well plates using an ABI PRISM 7900HT Sequence Detection System (PE Applied Biosystems, Foster City, CA, USA). TaqMan primers and probes (Table 2) were derived from the commercially available 'TaqMan Gene Expression Assays' (http://products.appliedbiosystems.com/). A keyword search for each gene name or accession number was performed, and the respective assay kit was ordered directly from the website. Amplification reactions were carried out in triplicate with $0.1 \mu \mathrm{l}$ template cDNA, $1 \mu \mathrm{l}$ TaqMan Universal PCR Master Mix (Applied Biosystems) and $1 \mu \mathrm{l}$ FAMlabeled Assay-on-Demand Gene expression Assay Mix (Applied Biosystems; Table 2). Thermal cycling was initiated with a $2 \mathrm{~min}$ incubation at $50{ }^{\circ} \mathrm{C}$ followed by a first denaturation step of $10 \mathrm{~min}$ at $95^{\circ} \mathrm{C}$, and then by $40-50$ cycles of $15 \mathrm{~s}$ at $95^{\circ} \mathrm{C}$ and $1 \mathrm{~min}$ at $60^{\circ} \mathrm{C}$. In each assay, a standard curve with six cDNA dilutions was recorded, and four no-template controls were included.

\section{Quantification and normalization}

Quantification was performed by the standard-curve method. In summary, a standard curve was prepared in each PCR assay for all genes using serial dilutions (1:1, $1: 3,1: 9,1: 18,1: 36$, and 1:72) of calibrator cDNA (rat reference RNA, Stratagene, La Jolla, CA, USA). The mean $C_{\mathrm{t}}$-value for triplicates was calculated, and the gene concentration (or gene copy number) of test samples was determined, based on standard curves. Expression from target genes was then normalized using the expression from an endogenous control gene.

\section{Statistical analysis}

The normalized expression levels were log transformed and the Welch's $t$-test was used to compare EAC with NME. For all samples, gene expressions are presented as fold changes, calculated as the ratio between the expression levels in tumor samples and the geometric mean of expression levels in the NME group.

\section{Al scoring at the Pten locus}

Analysis of AI at the Pten locus in the tumor material was performed by allelotyping using a polymorphic intragenic Pten marker (Sjöling et al. 2001) as well as four other markers (D1Rat79, D1Rat81, D1Rat169, and D1Rat300), located close to the Pten gene. Primer pair sequences for these markers were obtained from public databases. PCR amplification, scoring the AI and calculation of the allelic imbalance ratio (AIR) was performed essentially as described previously (Behboudi et al. 2001).

\section{Gene sequencings}

PCR primers were designed (Table 3) based on the available sequences in GenBank for amplification of the entire coding sequences of the Pten, Irfl, and Ctnnbl genes using tumor cDNA as template. The entire coding region of Pten consisting of $1212 \mathrm{bp}$ (NM_031606) and the entire coding sequence of Irf1 consisting of 987 bp (NM_012591) plus $195 \mathrm{bp}$ in $5^{\prime} \mathrm{UTR}$, and $617 \mathrm{bp}$ in the $3^{\prime} \mathrm{UTR}$ were amplified in single fragments. The entire coding sequence of the Ctnnbl gene is 2353 bp long (NM_053357) and was amplified in two separate overlapping segments: segment A, from nucleotide 2-1317 (1315 bp) and segment B from nucleotide 1202-2353 (1151 bp). PCR amplification products were purified using GFX PCR

Table 2 TaqMan assays used in quantitative real-time PCR

\begin{tabular}{|c|c|c|c|c|c|}
\hline Gene & Assay & $\begin{array}{l}\text { Accession } \\
\text { numbers }\end{array}$ & Exon boundary & Assay location & $\begin{array}{l}\text { Gene location } \\
\text { (characters) }\end{array}$ \\
\hline Pten & Rn00477208_m1 & NM_031606.1 & $4-5$ & nt 255 & $1 q 52$ \\
\hline Cdkn2b (P16) & Rn00676469_m1 & NM_031550.1 & - & nt 168 & $5 q 32$ \\
\hline E-cadherin (Cdh1) & Rn00580109_m1 & NM_031334.1 & $3-4$ & nt 526 & $19 q 12$ \\
\hline$\beta$-catenin (Ctnnb1) & Rn00584431_g1 & NM_053357.2 & $11-12$ & nt 1957 & $8 q 32$ \\
\hline Irf1 & Rn00561424_m1 & NM_012591 & $1-2$ & nt 189 & $10 q 22$ \\
\hline Erbb2 & Rn00690259_m1 & NM_017003.2 & $1-2$ & nt 186 & $10 q 32.1$ \\
\hline Tp53 & Rn00755717_m1 & NM_030989 & $8-9$ & nt 1175 & $10 q 24$ \\
\hline Rps9 & Rn01530912_g1 & NM_031108.2 & $3-4$ & nt 318 & $1 q 12$ \\
\hline
\end{tabular}


Table 3 Primers used for sequencing of the entire coding sequences of the Pten, Ctnnb1, and Irf1 genes

\begin{tabular}{|c|c|c|c|}
\hline Gene & Primer designation & Primer sequence $\left(5^{\prime}\right.$ to $\left.3^{\prime}\right)$ & Reference sequence \\
\hline \multirow[t]{4}{*}{ Pten } & exon1F & TGACAGCCATCATCAAAGAG & NM_031606 \\
\hline & exon6F & GGAGTAACTATTCCCAGTCAGAG & \\
\hline & exon7R & CTTTTTGAGCATCTTGTTCTGTT & \\
\hline & exon9R & TCAGACTTTTGTAATTTGTGAAT & \\
\hline \multirow[t]{6}{*}{ Ctnnb1 part $A$} & AF & CAATGGCTACTCAAGCTGACC & NM_053357.2 \\
\hline & $2 \mathrm{~F}$ & GCCATGTTCCCTGAGACACT & \\
\hline & $3 \mathrm{~F}$ & CGCACCATGCAGAATACAAA & \\
\hline & $4 \mathrm{~F}$ & TTGCTCAACAAAACAAACGTG & \\
\hline & $2 \mathrm{R}$ & GCATGCCCTCATCTAGTGTC & \\
\hline & AR & ACCATCATCTTGTTTTTGTAATTATTG & \\
\hline \multirow[t]{5}{*}{ Ctnnb1 part B } & $\mathrm{BF}$ & AAGGCCTCCTTGGGACTCTA & NM_053357.2 \\
\hline & $5 \mathrm{~F}$ & TTGGATTGATCCGAAACCTT & \\
\hline & $6 \mathrm{~F}$ & CCACTGTTTGTGCAGTTGCT & \\
\hline & $7 \mathrm{~F}$ & CCAATGGCTTGGAATGAGAC & \\
\hline & $\mathrm{BR}$ & TATCAAACCAGGCCAGCTGAT & \\
\hline \multirow[t]{8}{*}{ Irf1 } & $1 \mathrm{~F}$ & CGACGAAGGAGTAGGACGAG & NM_012591 \\
\hline & $2 \mathrm{~F}$ & CCGATACAAAGCTGGGGAAAAAG & \\
\hline & $3 F$ & TCGTCAGCAGCAGTCTCTCT & \\
\hline & $4 \mathrm{~F}$ & GGATTGGCGTTCTGAGGTTA & \\
\hline & $1 \mathrm{R}$ & TTTGTATCGGCCTGTGTGAA & \\
\hline & $2 \mathrm{R}$ & CTATCTCGATGTCCССTCCA & \\
\hline & $3 R$ & TGGTGAGACCCTCTCTAAGCA & \\
\hline & $4 \mathrm{R}$ & CAGAAATGTGGCAAAGTCCA & \\
\hline
\end{tabular}

$\mathrm{F}$, forward primer; $\mathrm{R}$, reverse primer.

DNA and Gel Band Purification Kit (Amersham Pharmacia Biotech). The purified fragments were subjected to sequencing using a second set of primers (Table 3) as described previously (Nordlander et al. 2007). Briefly, using ABI PRISM BigDye1 Terminator v1.1 Cycle Sequencing Kit (PE Applied Biosystems), the purified DNA fragments were subjected to sequencing according to the protocol provided by the manufacturer. Sequencing products were then denatured, cooled on ice and 1.5-1.8 $\mu \mathrm{l}$ of each sample was separated in a pre-warmed $5 \%$ denaturing polyacrylamide gel in 1xTBE buffer ( $89 \mathrm{mM}$ Tris/ $89 \mathrm{mM}$ boric acid/2 mM EDTA, $\mathrm{pH}$ 8.3) using an automated ABI Prism 377 Genescan Analyzer (PE Applied Biosystems). The sequence data obtained were subjected to BLAST searches (http://www.ncbi.nlm. nih.gov/BLAST/) for comparison with published sequences and inspected visually in the Sequencing Analysis software (PE Applied Biosystems).

\section{Results}

\section{Real-time quantification PCR}

Gene expressions of Pten, Ctnnb1, Tp53, P16, Cdh1, $E r b b 2$, and Irf1 were examined in a panel of 29 rat EAC and seven NME cell cultures. Rps 9 showed the lowest variations in $\Delta C_{\mathrm{t}}$ levels, and was expressed at similar levels in all samples regardless of the cell type (i.e. EAC or NME). Thus, Rps 9 was selected and used as an internal reference for normalization in the quantitative Real-time PCR analysis.

In all but two of the genes tested (i.e. Irfl and Tp53), the expression levels were lower in the EAC compared with the NME samples. The results revealed that Pten, $C d h 1, P 16, E r b b 2$, and Ctnnb1 differed significantly in expression levels (nominal $P$ value $<0.05$, Fig. 2, Table 4). In addition, when removing one outlier for Tp53 in the NME group, this gene also showed significant difference in expression level between the two sample groups $(P=0.001$, fold $=2.1)$.

\section{Allelic imbalance analysis and mutation sequencing of the Pten gene}

To investigate the status of Pten in the EAC susceptible rat model, the region encompassing this tumor suppressor gene (located at RNO1q52) was screened for indications of AI. The Pten intragenic marker D1Lev1 (Sjöling et al. 2002) is informative between the BDII and SPRD strain animals, but not between BDII and BN rats. The D1Lev1 marker was used in conjunction with another informative microsatellite marker (D1Rat81) for AI scoring of Pten locus in 
Table 4 Summary of molecular analysis of EACs developed in the BDII rat tumor model compared with different subgroups of human ECs

\begin{tabular}{|c|c|c|c|c|c|c|}
\hline \multirow[b]{2}{*}{ Gene alteration } & \multirow[b]{2}{*}{ Pathway } & \multicolumn{4}{|c|}{ Human endometrial carcinoma ${ }^{c}$} & \multirow[b]{2}{*}{$\begin{array}{l}\text { Endometrial carcinoma in } \\
\text { BDIl rats }^{d}\end{array}$} \\
\hline & & Type I & Type II & $\begin{array}{l}\text { Arised from } \\
\text { HNPCC }\end{array}$ & $\begin{array}{l}\text { Familial } \\
\text { site-specific }\end{array}$ & \\
\hline $\begin{array}{l}\text { Microsatellite } \\
\text { instability }\end{array}$ & MMR & $20-45 \%$ & $0-5 \%$ & $90-95 \%$ & $8-9 \%$ & $0-5 \%$ \\
\hline PTEN mutations & Akt & $50-80 \%$ & $10 \%$ & $60-85 \%$ & $\mathrm{~N}$ & $\begin{array}{l}\text { No mutation, but } 2.3 \text { fold } \\
\text { down-regulation }^{\text {a }}\end{array}$ \\
\hline$K$-RAS mutations & Akt & $10-30 \%$ & $0-5 \%$ & $10 \%$ & $\mathrm{~N}$ & No \\
\hline $\begin{array}{l}\text { ERBB2 } \\
\text { over-expression }\end{array}$ & Akt & $10-30 \%$ & $45-80 \%$ & $\mathrm{~N}$ & $\mathrm{~N}$ & $\begin{array}{l}\text { No, to the contrary } 4.5 \text { fold } \\
\text { down-regulation }^{\mathrm{a}}\end{array}$ \\
\hline TP53 mutations & P53 & $10-20 \%$ & $90 \%$ & Low & $\mathrm{N}$ & $67 \%$ mutations $^{\mathrm{a}, \mathrm{b}}$ \\
\hline$P 16$ inactivation & P53 & $10 \%$ & $40 \%$ & $\mathrm{~N}$ & $\mathrm{~N}$ & $\begin{array}{l}57 \% \text { deletion and } 28 \\
\quad \text { fold down-regulation }{ }^{\text {a }}\end{array}$ \\
\hline $\begin{array}{l}C D H 1 \text { down- } \\
\text { regulation }\end{array}$ & Wnt & $10-20 \%$ & $80-90 \%$ & $\mathrm{~N}$ & $\mathrm{~N}$ & Yes, 23 fold $^{a}$ \\
\hline CTNNB1 mutations & Wnt & $20 \%$ & $0-5 \%$ & $\mathrm{~N}$ & $\mathrm{~N}$ & $\begin{array}{l}\text { No, to the contrary } \\
2.2 \text { fold down-regulation }\end{array}$ \\
\hline
\end{tabular}

$\mathrm{N}$, not known or no information available.

apresent work.

${ }^{\mathrm{b}}$ After removing one outlier from the NME samples.

${ }^{\circ}$ Kariola et al. 2005, Lax 2007, Liu 2007, Ollikainen et al. 2005.

dBehboudi et al. 2001, Helou et al. 2001, Nordlander et al. 2005, Adamovic et al. 2008.

animals of the SPRD cross. AI analysis of the tumors developed in the BN cross was performed using D1Rat81 plus three additional informative markers, D1Rat169, D1Rat300, and D1Rat79, spanning a region of $6.5 \mathrm{cM}$ at the Pten locus. In the SPRD cross, among three tumors heterozygous in this region, one (RUT6) showed AI at Pten. In animals from the BN cross, two out of four heterozygous tumors at Pten, displayed AI (RUT12 and RUT30). Taken together, three out of seven informative tumors exhibited $\mathrm{AI} / \mathrm{LOH}$ at the Pten locus. Furthermore, cDNA of the entire coding region (1212 bp) of Pten was screened for mutations. As comparison, cDNA from normal tissue was subjected to sequencing as well. No mutation was detected anywhere in the coding sequence of the Pten gene in any of the samples.

\section{Mutation sequencing of the Ctnnb1 gene}

The cDNA sequence for Ctnnbl gene was divided into two parts, A and B, for PCR amplification. Furthermore, these segments were PCR amplified in smaller fragments of 400-500 bp for direct sequencing (Table 3). cDNA from the three parental strains (i.e. BDII, SPRD, and BN) and 14 EAC tumors were sequenced. A difference in nucleotide 1109 in exon 6 (GenBank-our data, C-T), compared with the available GenBank sequence for rat Ctnnbl mRNA (NM_053357) was found. The new data will cause codon 368 to be changed from Prolin (CCT) to Leucine (CTT). Since we found the same sequence in all the three parental strains, we believe that this sequence may be representative for an important subset of inbred rat strains. This finding is supported by the fact that both humans and mice have a Leucine, rather than a Prolin, at the corresponding position in the homologous proteins. We have submitted the sequence to GenBank. No further sequence aberrations or mutations were found.

\section{Sequencing the Irf1 gene}

The entire coding region of rat Irfl gene is $987 \mathrm{bp}$. Using primers $1 \mathrm{~L}$ and $1 \mathrm{R}$ (Table 3), a fragment of $1799 \mathrm{bp}$ of the Irfl gene was amplified from cDNA. This fragment consists of $195 \mathrm{bp}$ of the $5^{\prime} \mathrm{UTR}$, the entire coding sequence and $617 \mathrm{bp}$ of the $3^{\prime} \mathrm{UTR}$. Using a set of eight primers (Table 3), the fragment was fully sequenced in the tumors and in normal cDNA from the parental strains (i.e. BDII, BN, and SPRD). The sequence obtained for IrfI normal cDNA for each strain was identical, but they differed in six nucleotides compared with the available GenBank reference sequence (NM_012591). The differences were (GenBank-our data): nucleotides 324 (T-A), 325 (TA), 338 (T-C), and 398 (T-A) in the coding sequence, whereas the nucleotides $\mathrm{C}$ and $\mathrm{A}$, which we found at positions 1521 and 1530 respectively, both inside the 
$3^{\prime}$ UTR, seem to be missing in GenBank sequence. We have submitted the sequence to GenBank. The new data for nucleotides 324 and 325 will cause codon 43 to be changed from Leucine (UUG) to Lysine (AAG). Since we found the same sequence in all three parental strains, we believe that this sequence may be representative for an important subset of inbred rat strains. This is supported by the fact that both humans and mice have a Lysine, rather than a Leucine, at the corresponding position in the homologous proteins. The base substitutions at positions 338 and 398 represent third nucleotide changes in codons 47 and 67, respectively, and were silent, causing no amino acid substitution. Subsequently, Irfl was amplified and sequenced in 14 tumors samples. Three EAC tumors (RUT3, NUT4, NUT12) displayed variants in the gene sequence, compared with the normal cDNA sequence. RUT3 that contained two copies of proximal RNO10 (Nordlander et al. 2007) displayed a deletion of three nucleotides in exon 9 at codon 312 (1131-1133, NM_012591), resulting in deletion of one amino acid, Alanine, from the Irfl activator domain. Possibly, this change occurred because of nucleotide slippage, since the result of the mutation is that a double CTG triplet (CTGCTG) becomes a single CTG (Fig. 1A). The cDNA from NUT12 (showing two copies of proximal RNO10 (Nordlander et al. 2007)) displayed deletion of 146 nucleotides (nucleotides 349-495, NM_012591) encoding 50 amino acids (codon 49 to 99) of the Irfl DNA binding domain (Fig. 1B). The deletions resulted in the omission of 34 and 112 nucleotides from the end of exon 2 and the beginning of exon 3, respectively. Analyzing the deletion site, it was found that the break points occurred within a motif of seven nucleotides, AACAAGG, which was present in both exons in the intact allele, whereas in the deleted allele only one motif was retained. The third tumor (NUT4TC; 2-3 copies of proximal RNO10)

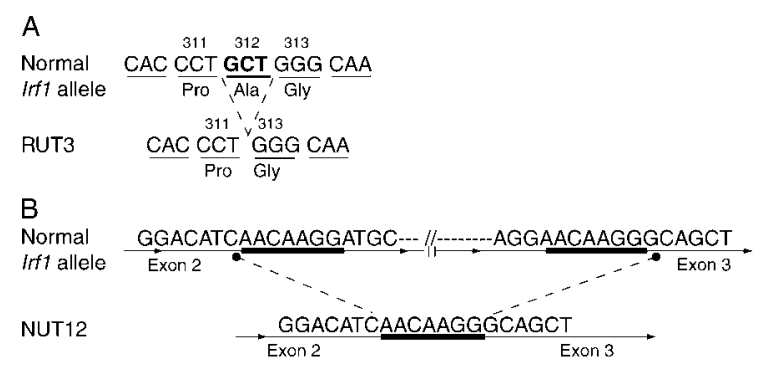

Figure 1 Irf1 transcript aberrations. (A) Deletion of Irf1 codon 312 in RUT3 (cDNA template); (B) Absence of $146 \mathrm{bp}$ in the Irf1 cDNA from NUT12. The lost segment lies between two identical 7-base sequences situated in exons 2 and 3 (cDNA template). displayed deletion of $318 \mathrm{bp}$ (nucleotides 1310-1628, NM_012591) from $3^{\prime}$ UTR of the gene. In summary, only three EAC samples showed sequence aberrations of the Irfl gene in this investigation, and the expression level did not differ significantly in the EAC versus the NME samples (Fig. 2).

\section{Discussion}

Human type I ECs are characterized by frequent microsatellite instability as well as PTEN, K-RAS, and CTNNB1 mutations, whereas TP53 mutation, P16 inactivation, over-expression of $E R B B 2$, and low $C D H 1$ expression are common features of type II tumors. EC arising in HNPCC are characterized by inherited mutation in the MMR genes, while molecular data are scarce for the familial site-specific subtype.

BDII rat spontaneous EC are largely similar to human type I tumors in their histology and cellular aspects of their pathogenesis (Vollmer 2003). Frequent Tp53 mutations (Nordlander et al. 2007) and lack of $K$-ras mutation (Helou et al. 2001) were shown in BDII rat EACs. Additionally, neither microsatellite instability (Behboudi et al. 2001, Adamovic et al. 2005, Nordlander et al. 2005) nor Erbb2 amplifications (Helou et al. 2001) were previously found among BDII rat tumors. Expression of Estrogen receptor $\alpha(E R I)$ was detected in the majority and progesterone receptor $(P g r)$ in a subset of these tumors (Karlsson et al. 2001). Here, we combined gene expression, AI, and gene mutation analysis to further characterize molecular features of endometrial carcinogenesis in the BDII rat model with respect to available molecular data for different subtypes of human ECs (Table 4).

The PTEN tumor suppressor gene has been described as one of the most frequently altered tumor suppressor genes in human cancer (Steck et al. 1997, Li \& Sun 1998, Planchon et al. 2008). PTEN exhibits combined $\mathrm{LOH}$ of one allele and mutation in the other allele at frequencies up to $50 \%$ in human endometrial tumors. PTEN mutations have even been detected in $33-55 \%$ of the endometrial precancerous lesions (Risinger et al. 1997, 1998, Tashiro et al. 1997, Konopka et al. 2002, Latta \& Chapman 2002). We found no sequence alterations in the entire coding region of the Pten gene in any of the BDII rat tumors. However, we found that AI at the Pten locus was present in about $50 \%$ of these tumors. We also found significant lower levels of mRNA (2.3 fold) in the EAC tumors compared with the NME samples. Taken together, these results suggest that Pten is likely involved in tumorigenesis of BDII rat EAC. Similar conclusions regarding Pten functioning in a 

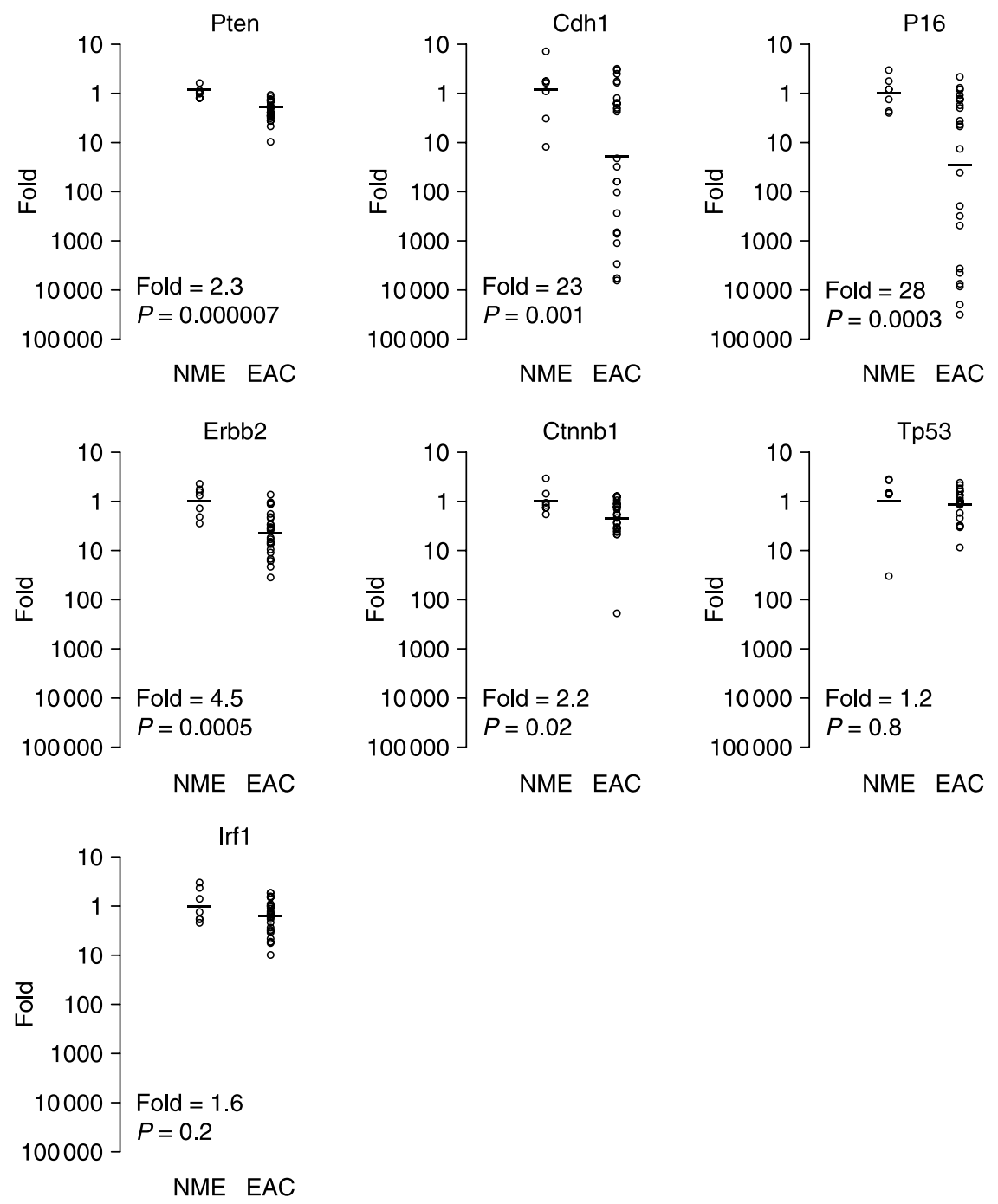

Figure 2 Real-time RT-PCR analysis of seven genes in a panel of 29 rat endometrial adenocarcinomas (EAC) compared with seven pre-malignant (NME) samples. Expression level of each gene in each sample is presented as fold change. Significant down regulation of Pten, Cdh1, P16, Erbb2, and Ctnnb1 was observed among EAC tumors, where no significant changes were detected for Tp53, and Irf1. For Tp53, when one outlier was removed from the NME sample group, this gene also showed significant difference in expression between EAC and NME samples $(P=0.001$, Fold $=2.1)$. NME, premalignant endometrium; EAC, rat $\mathrm{EAC}$ tumors; $P$, nominal $P$ value.

haploinsufficient mode has been drawn in several other reports (Di Cristofano et al. 1998, Kwabi-Addo et al. 2001, Sjöling et al. 2002, Kolasa et al. 2006, Kwon et al. 2008). Decreasing level of PTEN expression was shown to correlate with the progressive outcome of solid cancers, including ovarian, prostate, and cervical cancers (Harima et al. 2001, Jiang \& Liu 2008). Thus, in Pten inactivation, although through a different mechanism (i.e. haploinsufficiency/lower expression in BDII rat versus gene mutations in human), the BDII rat tumors can be related to human type I ECs.

CTNNB1 mutation is present in about $25-40 \%$ of human endometrioid (type I) carcinomas (Saegusa et al. 2001, Lax 2004). CTNNB1 is crucial in cell-cell adhesion through a complex with $C D H 1$. In addition, it is an important member of the WNT signal transduction pathway that is required for adult tissue maintenance (Logan \& Nusse 2004). The function of CTNNB1 in endometrioid tumorigenesis is still unknown; no correlation to microsatellite instability, $K-R A S, P T E N$, and/or CTNNB1 mutations have been found, suggesting that the WNT/CTNNB1 pathway may play an independent role in endometrial cancer tumorigenesis (Palacios et al. 2001). In the present work, no mutation in the Ctnnbl gene was found in BDII rat EAC tumors. In the Real-time PCR analysis, 
however, the expression level of Ctnnbl was found to be significantly lower (2.2-fold) in the EAC compared with the NME samples. ERBB2 oncogene codes for a transmembrane receptor tyrosine kinase involved in cell signaling. Over-expression add/or amplification of this gene seems to play a role in $10-30 \%$ of highergrade human ECs (Liu 2007). Neither Erbb2 amplification (Helou et al. 2001) nor over expression was found in BDII rat EAC tumors.

Cadherins are a family of adhesion molecules essential for tight connection between cells. E-Cadherin $(\mathrm{CDH1})$ is mainly expressed in epithelial cells. Reduced $\mathrm{CDHI}$ expression is associated with dysfunction of cell-cell adhesion system and has been shown to promote tumor invasion and metastasis (Strauli \& Haemmerli 1984, Volk et al. 1984). Decreased expression of $\mathrm{CDH} 1$ correlates with high histological grade and aggressive behavior and has been reported in 10-20\% of type I and in $80-90 \%$ of type II human endometrial tumors (reviewed in Lax (2004, 2007) and Liu (2007)). Here, we found Cdhl expressed to a significantly lower level in the EAC compared with the NME sample groups. Our results are congruent with previous reports on $\mathrm{CDH} 1$, and suggest a parallel expression pattern of the gene in the BDII EAC and human EC tumors.

$P 16$ is a tumor suppressor gene encoding for a cellcycle regulatory protein and inactivation of this gene is suggested to lead to cell anchorage independency, invasion, and metastasis. Loss of P16 expression has been found more commonly in human serous (type II, about $45 \%$ ) compared with endometrioid carcinomas (type I, less than 10\% and mainly found in high-grade tumors; Salvesen et al. 2000). P16 inactivation correlates with $K$-RAS mutations, high stage and high grading of tumors as well as poor survival (Salvesen et al. 2005). We found significant low expression level (28-fold) of P16 in the EAC tumors, which is in accordance to result from a recent study indicating frequent hemi- or homozygous deletions of $P 16$ in BDII rat EAC tumors (Adamovic et al. 2008). However, due to lack of $K$-ras mutations in this model, no correlation between inactivation of $P 16$ and mutation of $K$-ras in the BDII rat tumor model can be concluded.

Loss of IRFI has been reported to be a critical event in the development of human leukemia (Willman et al. 1993, Green et al. 1999). Additionally, frequent deletions in the IRFI locus have been detected in esophageal and gastric cancers (Ogasawara et al. 1996, Tamura et al. 1996). Several studies have suggested that post-transcriptional aberrations in the IRFI gene also may account for loss of IRFI function in tumors
(Green et al. 1999). In humans, the IRFI mRNA is expressed at a low basal level in a variety of cell types (Tanaka \& Taniguchi 2000, Kroger et al. 2002). In the uterus, the site of expression of IRFI has been localized to the glandular epithelial compartment of the endometrium (Jabbour et al. 1999), i.e. the cell types from which EAC arises. Down-regulation of IRF 1 is a constant finding in endometrial tumorigenesis (Giatromanolaki et al. 2004) and expression of IRFI has been shown to be the highest in normal endometrium, and decrease with the grade of endometrioid adenocarcinoma (Kuroboshi et al. 2003). Earlier, in AI analysis of RNO10 in the BDII rat tumors (Behboudi et al. 2001, Nordlander et al. 2005) we identified three segments in the proximal part of the chromosome, which were commonly affected by AI. Irfl is a tumor suppressor gene located at RNO10q22 (Behboudi et al. 2002) and thus was selected for further analysis. In the present work, however, we found that the Irfl expression in the rat EAC did not significantly differ from the NME samples. Additionally, we detected aberration/mutation in the coding region of the Irfl transcript in only three samples, suggesting that IrfI is of less importance in the development of EAC tumors in this rat model.

In human malignancies, the TP53 tumor suppressor gene is reported as the most commonly altered gene. The mutant p53 protein is non-functional, but resists degradation and accumulates in the cell. Mutations of the TP53 gene are a frequent and characteristic finding in type II serous and are considered to be an early event in tumorigenesis (Ryan et al. 2005). In type I tumors, abnormalities of Tp53 are suggested as late events and are thought to be important step in the progression to high-grade tumors (Ryan et al. 2005). Earlier, we found recurrent Tp53 mutations (67\%) in BDII rat EAC tumors (Nordlander et al. 2007). However, in majority of the mutations resulting in amino acid exchange, the hydrophobicity or hydrophilicity was maintained by equivalent substitutions. In the present work, we could show that $T p 53$ was expressed to a significantly lower level in the EAC than in the NME samples, only when one outlier in the NME group was removed from the analysis. Otherwise, there is no significant difference in expression between the two groups.

In conclusion, the EC developed in BDII rats are suggested to be related to human type I tumors, since they are hormone-related (Deerberg \& Kaspareit 1987, Deerberg et al. 1995), express Esrl (Karlsson et al. 2001) and histologically from endometrioid type (Kaspareit-Rittinghausen et al. 1987). At the molecular level, it has been shown that P16 deletions (Adamovic 
et al. 2008) and Tp53 mutations (Nordlander et al. 2007) are frequent, Furthermore, Erbb2 amplification, $K$-ras mutation (Helou et al. 2001), and microsatellite instability (Behboudi et al. 2001, Adamovic et al. 2005, Nordlander et al. 2005) were not common features of BDII rat EAC tumors. Here, we combined gene expression, AI and gene mutation analysis to further characterize molecular alterations of endometrial carcinogenesis in this tumor model with respect to available molecular data for different subtypes of human ECs. No mutation in Pten and Ctnnbl was found. In gene expression and AI analysis, we found recurrent AI at the Pten locus accompanied by lower expression of the gene, substantial down-regulation of $P 16$ and $C d h 1$ and slightly lower expression of Ctnnb1 and Tp53 in BDII rat EACs compared with the control samples (Table 4). In conclusion, our results suggest that endometrial carcinogenesis in BDII rats can be related to higher-grade human type I ECs.

It should be stressed that the rat disease genes identified so far have shown remarkable relevance to related human disease phenotypes (Aitman et al. 2008, Samuelson et al. 2008). Furthermore, there are instances that the rat model has inspired new therapeutic approaches (Gelderman et al. 2007, Pravenec \& Kurtz 2007). The efficiency of translating results from rat models to the human thus seems to be high and rat model studies should thus provide important insights into disease genetics and mechanisms, as well as new therapeutic approaches. The present molecular data suggest that spontaneous endometrial carcinogenesis in the BDII rat tumor model can essentially be related to higher-grade human type I endometrioid carcinomas. Thus, in experimental setups characterizing the molecular mechanisms of human type I tumors, testing and developing new substances for therapeutic approaches, and examining safety of new drugs, in particular, the endocrine active substances intended for use in tumor therapy, this model is of potentially high value and should be considered as one of the most important contributing in vivo tools currently available for research on this malignancy.

\section{Declaration of interest}

There is no conflict of interest that could be perceived as prejudicing the impartiality of the research reported.

\section{Funding}

This work was supported by The Swedish Cancer Society, The Gunvor and Ivan Svenssons Foundation. The Royal Physiographic Society in Lund (Nilsson-Ehle Foundation), The Assar Gabrielsson Foundation, The Wilhelm and
Martina Lundgren's Foundation, and The Adlerbertska Foundation.

\section{Acknowledgements}

We are grateful to Cecilia Börjesson and Dr Åsa Sjöling for their valuable contribution to this manuscript. The real-time PCR was performed at the Genomics Core Facility platform at the Sahlgrenska Academy, University of Gothenburg, which was funded by a grant from the Knut and Alice Wallenberg Foundation.

\section{References}

Adamovic T, Trossö F, Roshani L, Andersson L, Petersen G, Rajaei S, Helou K \& Levan G 2005 Oncogene amplification in the proximal part of chromosome 6 in rat endometrial adenocarcinomas as revealed by combined BAC/PAC FISH analysis, chromosome painting, zooFISH and allelotyping analysis. Genes, Chromosomes and Cancer 44 139-154.

Adamovic T, Hamta A, Roshani L, Lü X, Röhme D, Helou K, Klinga-Levan K \& Levan G 2008 Rearrangement and allelic imbalance on chromosome 5 leads to homozygous deletions in the CDKN2A/2B tumor suppressor gene region in rat endometrial cancer. Cancer Genetics and Cytogenetics 184 9-21.

Aitman TJ, Critser JK, Cuppen E, Dominiczak A, FernandezSuarez XM, Flint J, Gauguier D, Geurts AM, Gould M, Harris PC et al. 2008 Progress and prospects in rat genetics: a community view. Nature Genetics $\mathbf{4 0}$ 516-522.

Behboudi A, Levan G, Hedrich HJ \& Klinga-Levan K 2001 High-density marker loss of heterozygosity analysis of rat chromosome 10 in endometrial adenocarcinoma. Genes, Chromosomes and Cancer 32 330-341.

Behboudi A, Roshani L, Kost-Alimova M, Montelius-Alatalo K, Röhme D, Klinga-Levan K \& Ståhl F 2002 Detailed chromosomal and radiation hybrid mapping in the proximal part of rat chromosome 10 and gene order comparison with mouse and human. Mammalian Genome 13 302-309.

Carcangiu ML \& Chambers JT 1992 Uterine papillary serous carcinoma: a study on 108 cases with emphasis on the prognostic significance of associated endometrioid carcinoma, absence of invasion, and concomitant ovarian carcinoma. Gynecologic Oncology 47 298-305.

Catasus L, Machin P, Matias-Guiu X \& Prat J 1998 Microsatellite instability in endometrial carcinomas: clinicopathologic correlations in a series of 42 cases. Human Pathology 29 1160-1164.

Cavanagh D, Fiorica JV, Hoffman MS, Durfee J \& Nicosia SV 1999 Adenocarcinoma of the endometrium: an institutional review. Cancer Control 6 354-360.

Di Cristofano A, Pesce B, Cordon-Cardo C \& Pandolfi PP 1998 Pten is essential for embryonic development and tumour suppression. Nature Genetics 19 348-355. 
Deerberg F \& Kaspareit J 1987 Endometrial carcinoma in BDII/Han rats: model of a spontaneous hormonedependent tumor. Journal of the National Cancer Institute 78 1245-1251.

Deerberg F, Pohlmeyer G, Lörcher K \& Petrow V 1995 Total suppression of spontaneous endometrial carcinoma in BDII/ Han rats by melengestrol acetate. Oncology 52 319-325.

Esteller M, Xercavins J \& Reventos J 1999 Advances in the molecular genetics of endometrial cancer (review). Oncology Reports 6 1377-1382.

Gelderman KA, Hultqvist M, Olsson LM, Bauer K, Pizzolla A, Olofsson P \& Holmdahl R 2007 Rheumatoid arthritis: the role of reactive oxygen species in disease development and therapeutic strategies. Antioxidants \& Redox Signaling 9 1541-1567.

Giatromanolaki A, Koukourakis MI, Ritis K, Mimidis K \& Sivridis E 2004 Interferon regulatory factor-1 (IRF-1) suppression and derepression during endometrial tumorigenesis and cancer progression. Cytokine 26 164-168.

Green WB, Slovak ML, Chen IM, Pallavicini M, Hecht JL \& Willman CL 1999 Lack of IRF-1 expression in acute promyelocytic leukemia and in a subset of acute myeloid leukemias with del(5)(q31). Leukemia 13 1960-1971.

Gruber SB \& Thompson WD 1996 A population-based study of endometrial cancer and familial risk in younger women. Cancer and steroid hormone study group. Cancer Epidemiology, Biomarkers and Prevention 5 411-417.

Harima Y, Sawada S, Nagata K, Sougawa M, Ostapenko V \& Ohnishi T 2001 Mutation of the PTEN gene in advanced cervical cancer correlated with tumor progression and poor outcome after radiotherapy. International Journal of Oncology 18 493-497.

Hecht JL \& Mutter GL 2006 Molecular and pathologic aspects of endometrial carcinogenesis. Journal of Clinical Oncology 24 4783-4791.

Helou K, Walentinsson A, Beckmann B, Johansson A, Hedrich HJ, Szpirer C, Klinga-Levan K \& Levan G 2001 Analysis of genetic changes in rat endometrial carcinomas by means of comparative genome hybridization. Cancer Genetics and Cytogenetics 127 118-127.

Jabbour HN, Critchley HO, Yu-Lee LY \& Boddy SC 1999 Localization of interferon regulatory factor-1 (IRF-1) in nonpregnant human endometrium: expression of IRF-1 is up-regulated by prolactin during the secretory phase of the menstrual cycle. Journal of Clinical Endocrinology and Metabolism 84 4260-4265.

Jiang BH \& Liu LZ 2008 PI3K/PTEN signaling in tumorigenesis and angiogenesis. Biochimica et Biophysica Acta 1784 150-158.

Kariola R, Abdel-Rahman WM, Ollikainen M, Butzow R, Peltomäki P \& Nyström M 2005 APC and brta-catenin protein expression patters in HNPCC-related endometrial and colorectal cancers. Familial Cancer 4 187-190.

Karlsson A, Helou K, Walentinsson A, Hedrich HJ, Szpirer C \& Levan G 2001 Amplification of Mycn, Ddx1, Rrm2, and Odc1 in rat uterine endometrial carcinomas. Genes, Chromosomes and Cancer 31 345-356.
Karlsson S, Holmberg E, Askerlund A \& Levan KK 2007 Altered transforming growth factor-beta pathway expression pattern in rat endometrial cancer. Cancer Genetics and Cytogenetics 177 43-50.

Kaspareit-Rittinghausen J, Deerberg F \& Rapp K 1987 Mortality and incidence of spontaneous neoplasms in BDII/Han rats. Zeitschrift für Versuchstierkunde 30 209-216.

Kolasa IK, Rembiszewska A, Janiec-Jankowska A, Dansonka-Mieszkowska A, Lewandowska AM, Konopka B \& Kupryjańczyk J 2006 PTEN mutation, expression and $\mathrm{LOH}$ at its locus in ovarian carcinomas. Relation to TP53, K-RAS and BRCA1 mutations. Gynecologic Oncology 103 692-697.

Konopka B, Paszko Z, Janiec-Jankowska A \& Goluda M 2002 Assessment of the quality and frequency of mutations occurrence in PTEN gene in endometrial carcinomas and hyperplasias. Cancer Letters 178 43-51.

Kroger A, Koster M, Schroeder K, Hauser H \& Mueller PP 2002 Activities of IRF-1. Journal of Interferon \& Cytokine Research 22 5-14.

Kuroboshi H, Okubo T, Kitaya K, Nakayama T, Daikoku N, Fushiki S \& Honjo H 2003 Interferon regulatory factor-1 expression in human uterine endometrial carcinoma. Gynecologic Oncology 91 354-358.

Kwabi-Addo B, Giri D, Schmidt K, Podsypanina K, Parsons R, Greenberg N \& Ittmann M 2001 Haploinsufficiency of the Pten tumor suppressor gene promotes prostate cancer progression. PNAS 98 11563-11568.

Kwon CH, Zhao D, Chen J, Alcantara S, Li Y, Burns DK, Mason RP, Lee EY, Wu H \& Parada LF 2008 Pten haploinsufficiency accelerates formation of high-grade astrocytomas. Cancer Research 68 3286-3294.

Latta E \& Chapman WB 2002 PTEN mutations and evolving concepts in endometrial neoplasia. Current Opinion in Obstetrics and Gynecology 14 59-65.

Lax SF 2004 Molecular genetic pathways in various types of endometrial carcinoma: from a phenotypical to a molecular-based classification. Virchows Archiv 444 213-223.

Lax SF 2007 Molecular genetic changes in epithelial, stromal and mixed neoplasms of the endometrium. Pathology 39 46-54.

Li DM \& Sun H 1998 PTEN/MMAC1/TEP1 suppresses the tumorigenicity and induces G1 cell cycle arrest in human glioblastoma cells. PNAS 95 15406-15411.

Liu FS 2007 Molecular carcinogenesis of endometrial cancer. Taiwanese Journal of Obstetrics \& Gynecology 46 26-32.

Logan CY \& Nusse R 2004 The Wnt signaling pathway in development and disease. Annual Review of Cell and Developmental Biology 20 781-810.

Lynch HT \& de la Chapelle A 1999 Genetic susceptibility to non-polyposis colorectal cancer. Journal of Medical Genetics 36 801-818. 
Miyaki M, Konishi M, Tanaka K, Kikuchi-Yanoshita R, Muraoka M, Yasuno M, Igari T, Koike M, Chiba M \& Mori T 1997 Germline mutation of MSH6 as the cause of hereditary nonpolyposis colorectal cancer. Nature Genetics 17 271-272.

Nordlander C, Behboudi A, Levan G \& Klinga-Levan K 2005 Allelic imbalance on chromosome 10 in rat endometrial adenocarcinomas. Cancer Genetics and Cytogenetics 156 158-166.

Nordlander C, Karlsson S, Karlsson Å, Sjöling Å, Winnes M, Klinga-Levan K \& Behboudi A 2007 Analysis of chromosome 10 aberrations in rat endometrial cancer evidence for a tumor suppressor locus distal to Tp53. International Journal of Cancer 120 1472-1481.

Ogasawara S, Tamura G, Maesawa C, Suzuki Y, Ishida K, Satoh N, Uesugi N, Saito K \& Satodate R 1996 Common deleted region on the long arm of chromosome 5 in esophageal carcinoma. Gastroenterology 110 52-57.

Ollikainen M, Abdel-Rahman WM, Lindroos A, Kariola R, Järvelä I, Pöyhönen M, Butzow R \& Peltomäki P 2005 Molecular analysis of familial endometrial carcinoma: a manifestation of hereditary nonpolyposis colorectal cancer or a separate syndrome? Journal of Clinical Endocrinology 23 4609-4616.

Palacios J, Catasús L, Moreno-Bueno G, Matias-Guiu X, Prat J \& Gamallo C 2001 Beta- and gamma-catenin expression in endometrial carcinoma. Relationship with clinicopathological features and microsatellite instability. Virchows Archiv 438 464-469.

Parc YR, Halling KC, Burgart LJ, McDonnell SK, Schaid DJ, Thibodeau SN \& Halling AC 2000 Microsatellite instability and hMLH1/hMSH2 expression in young endometrial carcinoma patients: associations with family history and histopathology. International Journal of Cancer 86 60-66.

Peltomaki P \& Vasen HF 1997 The International Collaborative Group on HNPCC. Mutations predisposing to hereditary nonpolyposis colorectal cancer: database and results of a collaborative study. Gastroenterology 113 1146-1158.

Planchon SM, Waite KA \& Eng C 2008 The nuclear affairs of PTEN. Journal of Cell Science 121 249-253.

Pravenec M \& Kurtz TW 2007 Molecular genetics of experimental hypertension and the metabolic syndrome: from gene pathways to new therapies. Hypertension 49 941-952.

Risinger JI, Hayes AK, Berchuck A \& Barrett JC 1997 PTEN/MMAC1 mutations in endometrial cancers. Cancer Research 57 4736-4738.

Risinger JI, Hayes K, Maxwell GL, Carney ME, Dodge RK, Barrett JC \& Berchuck A 1998 PTEN mutation in endometrial cancers is associated with favorable clinical and pathologic characteristics. Clinical Cancer Research 4 3005-3010.

Roshani L, Wedekind D, Szpirer J, Taib Z, Szpirer C, Beckman B, Rivière M, Hedrich HJ \& Klinga-Levan K 2001 Genetic identification of multiple susceptibility genes involved in the development of endometrial carcinoma in a rat model. International Journal of Cancer 94 795-799.

Roshani L, Mallon P, Sjostrand E, Wedekind D, Szpirer J, Szpirer C, Hedrich HJ \& Klinga-Levan K 2005 Genetic analysis of susceptibility to endometrial adenocarcinoma in the BDII rat model. Cancer Genetics and Cytogenetics 158 137-141.

Ryan AJ, Susil B, Jobling TW \& Oehler MK 2005

Endometrial cancer. Cell and Tissue Research 322 53-61.

Saegusa M, Hashimura M, Yoshida T \& Okayasu I 2001

Beta-catenin mutations and aberrant nuclear expression during endometrial tumorigenesis. British Journal of Cancer 84 209-217.

Salvesen HB, Das S \& Akslen LA 2000 Loss of nuclear p16 protein expression is not associated with promoter methylation but defines a subgroups of aggressive endometrial carcinomas wiht poor prognosis. Clinical Cancer Research 6 153-159.

Salvesen HB, Kumar R \& Stefansson I 2005 Low frequency of BRAF and CDKN2A mutations in endometrial cancer. International Journal of Cancer 115 930-934.

Samuelson E, Levan K, Adamovic T, Levan G \& Horvath G 2008 Recurrent gene amplifications in human type I endometrial adenocarcinoma detected by fluorescence in situ hybridization. Cancer Genetics and Cytogenetics 181 25-30.

Sandles LG 1998 Familial endometrial adenocarcinoma. Clinical Obstetrics and Gynecology 41 167-171.

Sandles LG, Shulman LP, Elias S, Photopulos GJ, Smiley LM, Posten WM \& Simpson JL 1992 Endometrial adenocarcinoma: genetic analysis suggesting heritable site-specific uterine cancer. Gynecologic Oncology 47 167-171.

Sjöling Å, Lindblom H, Samuelson E, Yamasaki Y, Watanabe TK, Tanigami A \& Levan G 2001 Analysis of chromosomal aberrations involving chromosome 1q31q53 in a DMBA-induced rat fibrosarcoma cell line: amplification and overexpression of Jak2. Cytogenetics and Cell Genetics 95 202-209.

Sjöling A, Samuelson E, Adamovic T, Behboudi A, Röhme D \& Levan G 2002 Recurrent allelic imbalance at the rat Pten locus in DMBA-induced fibrosarcomas. Genes, Chromosomes and Cancer 36 70-79.

Steck PA, Pershouse MA, Jasser SA, Yung WK, Lin H, Ligon AH, Langford LA, Baumgard ML, Hattier T, Davis T et al. 1997 Identification of a candidate tumour suppressor gene, MMAC1, at chromosome 10q23.3 that is mutated in multiple advanced cancers. Nature Genetics 15 356-362.

Strauli P \& Haemmerli G 1984 The role of cancer cell motility in invasion. Cancer and Metastasis Reviews $\mathbf{3}$ 127-141.

Tamura G, Ogasawara S, Nishizuka S, Sakata K, Maesawa C, Suzuki Y, Terashima M, Saito K \& Satodate R 1996 Two distinct regions of deletion on the long arm of chromosome 5 in differentiated adenocarcinomas of the stomach. Cancer Research 56 612-615. 
Tanaka N \& Taniguchi T 2000 The interferon regulatory factors and oncogenesis. Seminars in Cancer Biology 10 73-81.

Tashiro H, Blazes MS, Wu R, Cho KR, Bose S, Wang SI, Li J, Parsons R \& Ellenson LH 1997 Mutations in PTEN are frequent in endometrial carcinoma but rare in other common gynecological malignancies. Cancer Research 57 3935-3940.

Volk T, Geiger B \& Raz A 1984 Motility and adhesive properties of high and low-metastatic murine neoplastic cells. Cancer Research 44 811-824.
Vollmer G 2003 Endometrial cancer: experimental models useful for studies on molecular aspects of endometrial cancer and carcinogenesis. Endocrine-Related Cancer 10 23-42.

Willman CL, Sever CE, Pallavicini MG, Harada H, Tanaka N, Slovak ML, Yamamoto H, Harada K, Meeker TC, List AF et al. 1993 Deletion of IRF-1, mapping to chromosome 5q31.1, in human leukemia and preleukemic myelodysplasia. Science 259 968-971. 